\title{
Mutación del gen KRAS en el cáncer de colon y recto
}

\author{
IVÁN ROA ${ }^{1}$, TAMARA SÁNCHEZ ${ }^{2}$, \\ ALEJANDRO MAJLIS ${ }^{3}$, KURT SCHALPER ${ }^{2}$
}

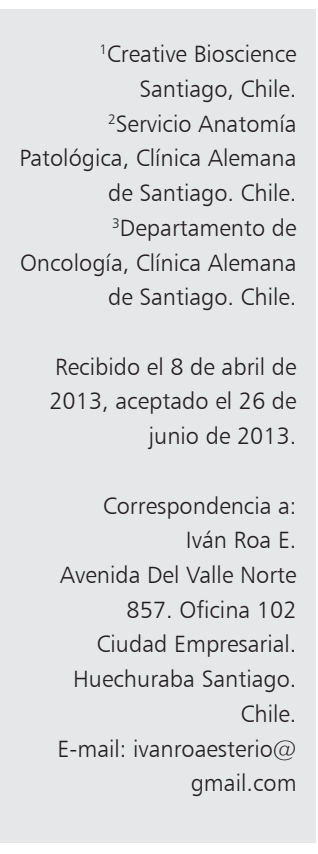

\section{KRAS gene mutation in colorectal cancer}

\begin{abstract}
Background: KRAS oncogene is involved in colorectal carcinogenesis in 22 to $45 \%$ of cases. Aim: To determine the frequency, types and distribution of KRAS mutations in colorectal cancer. Material and Methods: KRAS mutations studies were carried out in primary tumors and metastases of colo-rectal cancer from 56 women aged 60 \pm 14 years and 53 men aged $61 \pm 11$ years. Formalin fixed and paraffin embedded tissue samples were evaluated using RFLP (Restriction Fragment Length Polymorphism) and direct sequencing. Results: Primary tumors were located in the colon and rectum in $82(75.2 \%)$ and 24 cases (20\%), respectively. In three cases the extraction site of the tumor sample was unknown. In 46 cases (42.2\%) KRAS mutations were demonstrated. The main point mutations were located in codon 12 (80.4\%), G12D (39.1\%), G12V (24.2\%), G12S (6.5\%), G12A (4.3\%); G12C (4.3\%), G12R (2.1\%) and $19.6 \%$ at codon 13 (G13D). No differences were demonstrated in the frequency and distribution of mutations by gender, age, primary versus metastatic tumors or tumor location. Conclusions: In this series, $42 \%$ of colorectal cancer tissue samples had KRAS mutations. Their frequency and distribution are similar to those reported in the literature, except for $\mathrm{G} 12 \mathrm{C}$ mutation.
\end{abstract}

(Rev Med Chile 2013; 141: 1166-1172).

Key words: Colonic neoplasms; Rectal neoplasms; Sarcospan (Kras oncogeneassociated gene) protein, human.
E 1 cáncer de colon y recto es una causa importante de mortalidad por tumor maligno que afecta a la población chilena ${ }^{1,2}$. Su importancia desde el punto de vista epidemiológico y clínico-quirúrgico ya ha sido extensamente documentada en nuestro medio ${ }^{3,4}$. El cáncer de colon y recto es una de las neoplasias malignas que ha sido mejor caracterizada desde el punto de vista genético-molecular tanto en sus formas esporádicas como las hereditarias poliposas y no poliposas $^{5-7}$. Estos estudios han determinado la participación de múltiples genes y vías metabólicas en el crecimiento y desarrollo de este cáncer ${ }^{8-10}$. Algunas de estas vías han podido ser estimuladas o inhibidas mediante moléculas como anticuerpos monoclonales, lo que ha permitido establecer terapias dirigidas a blancos específicos, como es el caso de la vía del EGFr ${ }^{11-15}$. En la actualidad son centenares de trabajos en los que se han determinado el status de algunos de estos genes como EGFr, KRAS, BRAK, PIP3K, etc. ${ }^{16-18}$, siendo su estudio rutinario en la evaluación de pacientes con cáncer colo-rectal en la mayoría de los centros extranjeros $\mathrm{y}$ en algunos centros privados nacionales ${ }^{12,19,20}$. A pesar de esta realidad, los estudios a nivel genético y molecular en el cáncer de colon y recto en nuestro medio, son recientes y escasos ${ }^{21-27}$.

La familia de los genes RAS (HRAS, NRAS y KRAS) es uno de los grupos de oncogenes más frecuentemente alterados en las neoplasias humanas $^{28}$. El oncogén KRAS se localiza en el cromosoma 12 y participa en señalización de las vías PI3K/ PTEN/AKT y RAF/MEK/ERK ${ }^{7,29}$. Las proteínas codificadas por estos genes constituyen una estruc- 
tura proteica de $21 \mathrm{Kd}$ (p21), la que posee actividad GTP-asa, actuando en la vía de transducción de señales de crecimiento y diferenciación celular ${ }^{30}$. La mutación de este gen es el evento genético más comúnmente observado en el desarrollo de tumores en el ser humano (pulmón 30\%, colon $40 \%$, páncreas $80 \%$, tiroides $55 \%$, etc. $)^{31}$. Alrededor de 90\% de las mutaciones de este gen se localizan en sitios específicos del primer exón (codón 12 en aproximadamente $80 \%$ y con menor frecuencia en él codón 13 en alrededor de $15-20 \%)^{28,32}$. En el segundo exón se localiza el codón 61 con una frecuencia de mutación menor de 3\%. La mutación más frecuente ocurre en el segundo nucleótido del codón 12 y corresponden predominantemente a transiciones (guanina-adenina) con sustitución de ácido aspártico por glicina.

En el cáncer colorrectal se ha encontrado mutación del gen KRAS entre 30 y 50\% de los $\operatorname{casos}^{28,32,33}$. La actual terapia de algunos estadios avanzados de colon y recto incluyen el uso de anticuerpos monoclonales (panitumumab, cetuximab), capaces de bloquear la activación del $\mathrm{EGFr}^{34-36}$. En los pacientes no respondedores a esta terapia se demostró que las células tumorales eran portadoras de una de las mutaciones activantes del gen KRAS que se encuentra localizado corriente abajo en la vía del gen EGFr, lo que producía la activación de esta vía independiente del bloqueo del EGFr ${ }^{37,38}$. También fue demostrado que la determinación del estado silvestre (no mutado) o mutado del gen KRAS era capaz de predecir la respuesta del tumor al uso de inhibidores del EGRr, siendo su determinación clínicamente útil $^{13,39,40}$. Algunos estudios han demostrado que algunos tipos específicos de mutación de KRAS tienen relación con la sobrevida, como la mutación G12V, la que se asociaría a un pronóstico más adverso de la enfermedad en relación a otros tipos de mutaciones ${ }^{41-43}$. A pesar de la importancia y al fácil estudio de la estructura de este gen, no existe información publicada en nuestro medio respecto de la frecuencia y tipo de mutaciones del gen KRAS en Chile en el cáncer de colon y recto, por lo cual, desconocemos no sólo su frecuencia y el tipo de mutaciones presentes en nuestra población, lo cual, pudiese tener importancia terapéutica.

El objetivo de este trabajo es determinar la frecuencia y distribución de las mutaciones del gen KRAS en el cáncer de colon y recto (codones 12 y 13) en nuestro país.

\section{Material y Método}

\section{Casos}

Se incluyen en este estudio de muestras de 109 adenocarcinomas primarios en estadios incipientes, avanzados y metástasis de adenocarcinomas de colon y recto. A las muestras provenientes de tejidos fijados en formalina e incluidos en parafina (a las cuales) se les realizó estudio de mutación del gen KRAS en el Servicio de Anatomía Patológica de Clínica Alemana de Santiago entre los años 2008 y 2012 y en el Centro de Educación Médica e Investigaciones Clínicas CEMIC, Argentina, en el año 2008. Se confeccionó una base de datos anónima de las muestras, en la cual fueron consignados exclusivamente los siguientes parámetros: género, edad y localización del tumor.

\section{Protocolo de extracción, cuantificación y amplificación de ADN}

En los cortes seleccionados que incluían áreas con una población de al menos 30\% de células tumorales se realizó la desparafinización en baños de xilol e hidratación en concentraciones decrecientes de alcoholes hasta el agua y mantención de las muestras en solución tamponada. Posteriormente, se realizó disección de las áreas seleccionadas. Los fragmentos obtenidos fueron resuspendidos y centrifugados por $5 \mathrm{~min}$ a $13.000 \mathrm{rpm}$. La extracción de ADN se realizó utilizando el kit Axy Prep Multisource Genomic DNA Miniprep kit Axygen ${ }^{\circledR}$ de acuerdo a las instrucciones del fabricante. Se realizó determinación de la integridad del ADN, a través de la amplificación del gen de la $\beta$-globina con productos de amplificación esperados de 110 y 268pb. La mezcla utilizada para la reacción contenía: $0,2 \mathrm{mM}$ de dNTP, 1,5 mM de Cloruro de Magnesio, 12,5 pmol de cada partidor, $1 \mathrm{U}$ de Taq polimerasa (Gotaq Flexi DNA polymerase Promega ${ }^{\circledR}$ ) y $5 \mu \mathrm{l}$ de ADN en estudio, en un volumen de reacción de $50 \mu \mathrm{l}$. La verificación de los productos se realizó por electroforesis en geles de agarosa a $2 \%$.

\section{Amplificación exón 1 gen KRAS}

Las condiciones de la amplificación se realizó en similares condiciones y concentraciones que en el protocolo antes descrito de acuerdo a protocolos previamente reportados ${ }^{44}$. Se utilizaron los siguientes partidores del exón 1 con productos 
esperados de 122 pb: KRAS forward 5'GGC CTG CTG AAA ATG ACT GA 3' y KRAS reverse 3'TCT ATT GTT GGA TCA TAT TCG T5'.

\section{RFLP (Restriction Fragment Length Polimorphism)}

Se realizó PCR semianidado con los siguientes partidores: codón 12F AAA CTT GTG GTA GTT GGCCCT y codón 13F 5'GCCTGCTGAAAATGACTGAA con un producto esperado de $98 \mathrm{pb}$ y $96 \mathrm{pb}$ respectivamente. La digestión se realizó con enzimas de restricción BstNI para el sitio de corte del codón el codón 12 y Bgl I para el del codón 13 , con formación de fragmentos de $75 \mathrm{pb} / 23 \mathrm{pb}$ y $70 \mathrm{pb} / 26 \mathrm{pb}$ respectivamente. La visualización se realizó en geles de agarosa a 3\% teñidos con bromuro de etidio (Figura 1a).

\section{Secuenciación}

Los productos amplificados fueron purificados en columnas Qiagen, (Quiaquick. PCR purification kit) y junto con el partidor reverso fueron secuenciados en equipo ABI PRISM 3100 Applied Biosystems (Figura 1b).

\section{Controles}

Como control positivo, se empleó ADN de carcinomas de páncreas en los que previamente se había demostrado la mutación del KRAS en el codón 12. Como control negativo se usó ADN genómico comercial (Promega) y como control blanco se sustituyó el ADN por agua deionizada.

\section{Análisis estadístico}

Se realizó mediante test de $\chi^{2}$ y exacto de Fisher
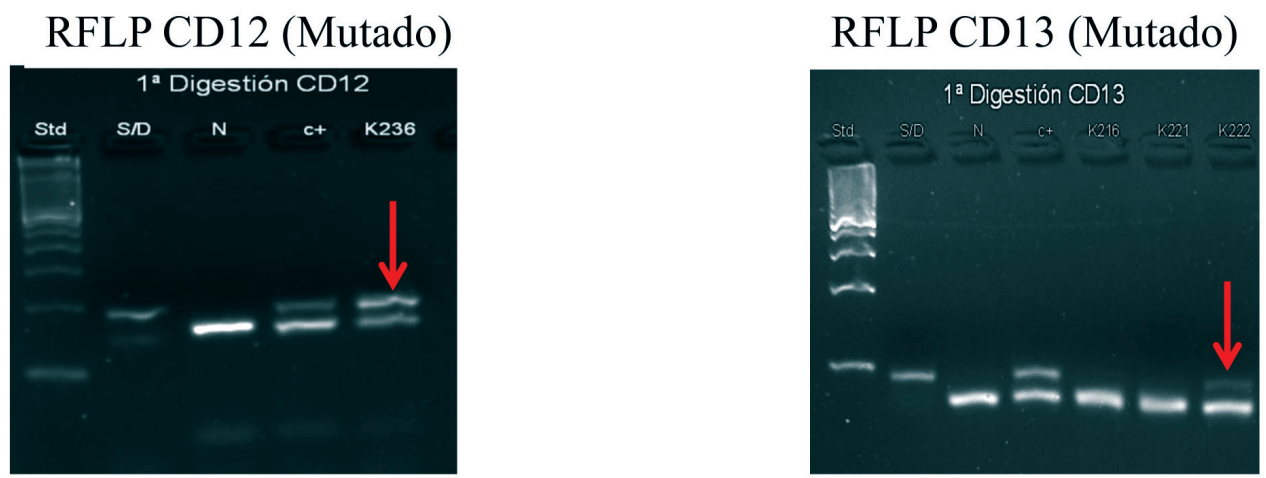

\section{Electroferograma mutación CD12}

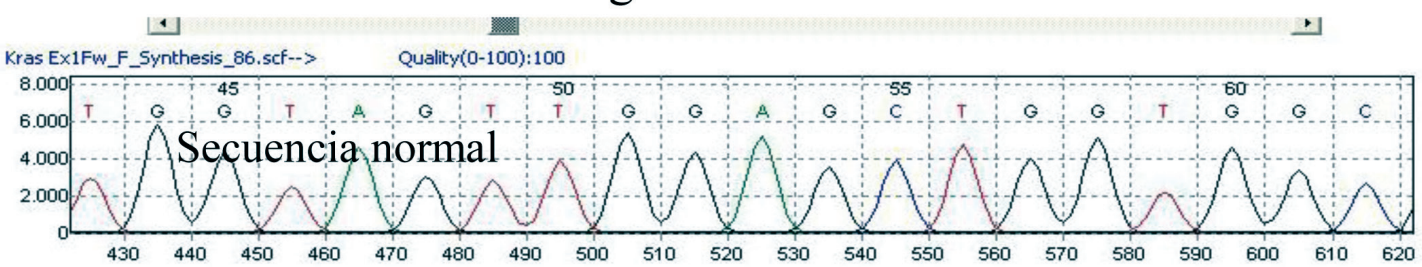

1673-IVAN-KB247-KB-EX1FWD-2012-06-13-B11.ab1--> Quality(0-100):38

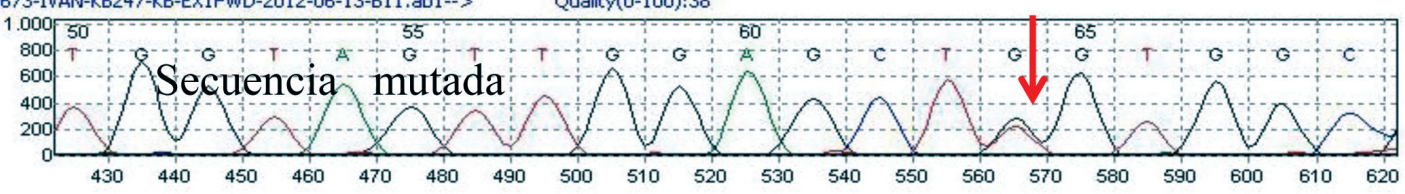

Figura 1. Detección de mutaciones del gen KRAS codones 12 y 13. 
Mutación gen KRAS en cáncer de colorrectal - I. Roa et al

para las tablas de contingencia y t-test para los promedios de muestras independientes.

\section{Resultados}

El grupo total estaba constituido por 109 casos, 56 casos $(51,3 \%)$ correspondieron a mujeres con un promedio de edad de 60,1 años (DS $\pm 13,7$ años) y los restantes 53 casos a hombres con un promedio de edad de 61,5 años (DS $\pm 10,7$ años). La totalidad de los casos correspondieron a adenocarcinomas. Los tumores primarios se localizaron en colon en 82 casos $(75,2 \%)$ y en recto en 24 casos (22\%). No se logró precisar la localización del tumor en 3 casos (2,8\%). En 46 casos (42,2\%), se demostró mutación del gen KRAS, los 63 casos restantes fueron de tipo silvestre o no mutado. Las mutaciones comprometieron al codón 12 en 37 casos $(80,4 \%)$ y al codón 13 en 9 casos $(19,6 \%)$. No se observaron diferencias entre la frecuencia de mutaciones de KRAS y el género e los pacientes. La distribución entre la edad y la frecuencia de mutaciones en relación al número de casos estudiados por década se resume en la Tabla 1. La mayor frecuencia de mutaciones se observó entre los 40 y 49 años (64,2\% de los casos examinados), sin embargo, la diferencia en la frecuencia de mutaciones entre los mayores y menores de 50 años no fue significativa $(\mathrm{p}=0,06)$.

La frecuencia de mutaciones en los tumores primarios fue mayor que las observadas en las metástasis ( $42,3 \%$ versus $33,3 \%$ ), sin embargo, esta diferencia no fue significativa tal y como ha sido reportado en series internacionales en las cuales esta diferencia es aún menor ${ }^{45,46}$.

Tabla 1. Frecuencia de mutaciones KRAS por edad

\begin{tabular}{|lccrc|}
\hline Edad & Mutado & No mutado & Total & $\%$ \\
\hline $20-29$ & 1 & 1 & 2 & 50,0 \\
\hline $30-39$ & 0 & 3 & 3 & 0,0 \\
\hline $40-49$ & 9 & 5 & 14 & 64,2 \\
\hline $50-59$ & 11 & 17 & 28 & 39,3 \\
\hline $60-69$ & 11 & 23 & 34 & 32,5 \\
$70-79$ & 12 & 12 & 24 & 50,0 \\
\hline$>80$ & 2 & 2 & 4 & 50,0 \\
\hline & 46 & 63 & 109 & 42,2 \\
\hline
\end{tabular}

La frecuencia de mutación, tipo de sustitución de base y cambio de aminoácido se resume en la Tabla 2, en la cual además, se compara con algunas series publicadas.

Las mutaciones más frecuentes fueron la G12D (aspartato) $(39,1 \%)$ y la G12V (valina) $(24,2 \%)$, le siguió en frecuencia la mutación del codón 13 (19,5\%), que en todos los casos correspondió a la G13D (aspartato) (Figura 2).

\section{Discusión}

Nuestros hallazgos muestran la presencia de mutación del gen KRAS en $42 \%$ de los cánceres de colon y recto, cifra muy similar a la reportada en los múltiples trabajos publicados en la literatura $^{32,33,41}$. Un hecho similar se observa en relación a la distribución porcentual de los distintos tipos de mutaciones, con sobre $80 \%$ de mutaciones del codón 12 y aproximadamente $20 \%$ en el codón 13. Sería recomendable ampliar el estudio a otros codones de menor frecuencia como el codón 61 y 146 , etc., a fin de precisar su exacta frecuencia y distribución en nuestra población. La distribución de los distintos tipos de mutaciones observadas, también se asemeja a lo reportado en la literatura. Las mutaciones G12D, G12V y G13D darían cuenta de $82,8 \%$ del total), siendo la mutación G12C (cisteína), de baja frecuencia en nuestro medio $(4,3 \%)$ en relación a la reportada en otras latitudes (entre $8 \%$ y $11,5 \%$ ) y cuyo significado debería ser estudiado ${ }^{45}$.

$\mathrm{Al}$ igual que en otras series no se observaron diferencias en la frecuencia de mutación de KRAS y género, localización (colon o recto) y edad de los pacientes, así como tumor primario o metástasis ${ }^{33}$. A pesar de observar una mayor frecuencia de mutaciones en pacientes menores de 50 años, esta diferencia no fue estadísticamente significativa. De esta manera, podríamos asumir que nuestros resultados traducen una realidad muy similar a otros grupos étnicos y poblacionales de otras latitudes.

Por lo tanto, la utilización de la determinación del status del gen KRAS para predecir la respuesta al tratamiento con anticuerpos monoclonales contra EGFr, tendría la misma connotación, es decir, en alrededor de $42 \%$ de nuestros pacientes no tendría indicación terapéutica. A este grupo se le debe agregar el grupo de pacientes con la mutación de BRAF, la cual es mutuamente excluyente de KRAS, y que también tiene la propiedad 


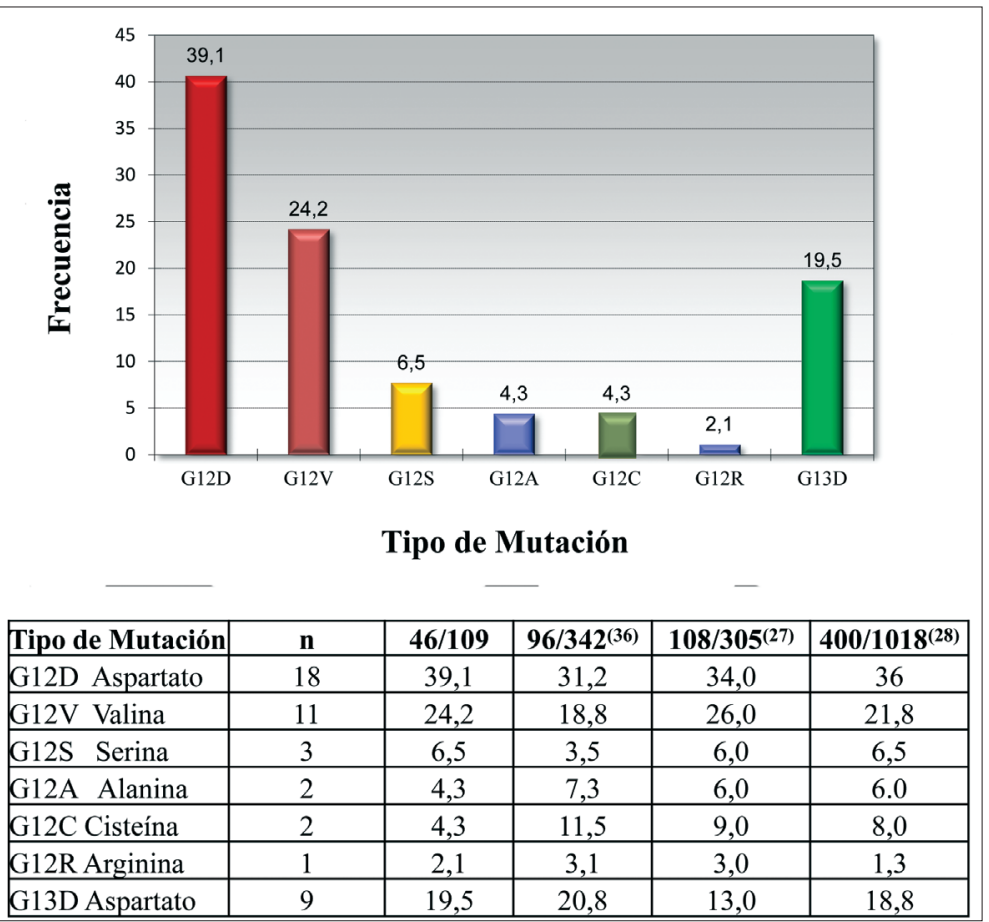

Figura 2. Tipo de mutación KRAS C 12 y 13. de mantener activa la vía del EGFr. Para este gen nuestros estudios preliminares (Roa, datos no publicados), en casos de pacientes con KRAS no mutado, muestran una frecuencia de mutación de BRAF que podría alcanzar sobre $15 \%$, lo que restringiría aún más el uso de bloqueadores o antagonistas del EGFr en el tratamiento del cáncer de colon y recto en nuestro país.

Respecto de las técnicas empleadas en este estudio, podemos señalar que el RFLP fue realizado siempre previo a la secuenciación y en todos, excepto un caso, hubo discordancia con este último método producto de un polimorfismo, por lo cual pudiese ser incorporada en nuestro medio como una técnica de tamizaje, tanto en centros asistenciales públicos y privados dada su sencillez y facilidad de interpretación ${ }^{44}$.

\section{Referencias}

1. http://deis.minsal.cl/index.asp. 2012.

2. Zarate AJ, Alonso FT, Garmendia ML, López-Kostner F. Increasing crude and adjusted mortality rates for colorectal cancer in a developing South American country. Colorectal Dis 2013; 15 (1): 47-51.

3. Donoso A, Villarroel L, Pinedo G. [Increase in colon cancer mortality rates in Chile, during the period 19902003]. Rev Med Chile 2006; 134 (2): 152-8.

4. Bannura G, Contreras J, Cumsille MA, García C, Portalier P. [Results of the surgical treatment of colorectal cancer: analysis of recurrence and survival in 400 patients]. Rev Med Chile 1995; 123 (4): 464-72.

5. Bacolod MD, Barany F. Molecular profiling of colon tumors: the search for clinically relevant biomarkers of progression, prognosis, therapeutics, and predisposition. Ann Surg Oncol 2011; 18 (13): 3694-700.

6. Fearon ER. Molecular genetics of colorectal cancer. Annu Rev Pathol 2011; 6: 479-507.

7. Wee S, Jagani Z, Xiang KX, Loo A, Dorsch M, Yao YM, et al. PI3K pathway activation mediates resistance to MEK inhibitors in KRAS mutant cancers. Cancer Res 2009; 69 (10): 4286-93.

8. Beerenwinkel N, Antal T, Dingli D, Traulsen A, Kinzler KW, Velculescu VE, et al. Genetic progression and the waiting time to cancer PLoS Comput Biol 2007; 3 (11): e225.

9. Frattini M, Balestra D, Suardi S, Oggionni M, Alberici P, Radice P, et al. Different genetic features associated with colon and rectal carcinogenesis. Clin Cancer Res 2004; 10 (12 Pt 1): 4015-21.

10. Jones S, Chen WD, Parmigiani G, Diehl F, Beerenwinkel $\mathrm{N}$, Antal T, et al. Comparative lesion sequencing provi- 
des insights into tumor evolution. Proc Natl Acad Sci U S A 2008; 105 (11): 4283-8.

11. Siddiqui AD, Piperdi B. KRAS mutation in colon cancer: a marker of resistance to EGFR-I therapy. Ann Surg Oncol 2010; 17 (4): 1168-76.

12. Dietel $M$, Jährens K, Laffert $M$, Hummel $M$, Blaker $H$, Múller BM, et al. Predictive molecular pathology and its role in targeted cancer therapy: a review focussing on clinical relevance. Cancer Gene Ther 2013.

13. Dahabreh IJ, Terasawa T, Castaldi PJ, Trikalinos TA. Systematic review: Anti-epidermal growth factor receptor treatment effect modification by KRAS mutations in advanced colorectal cancer. Ann Intern Med 2011; 154 (1):37-49.

14. Mendelsohn J. Targeting the epidermal growth factor receptor for cancer therapy. J Clin Oncol 2002; 20 (18 Suppl): 1S-13S.

15. Heinemann V, Douillard JY, Ducreux M, Peeters M. Targeted therapy in metastatic colorectal cancer - An example of personalised medicine in action. Cancer Treat Rev 2013.

16. Brandi G, Tavolari S, De Rosa F, Di Girolamo S, Agostini V, Barbera MA, et al. Antitumoral efficacy of the protease inhibitor gabexate mesilate in colon cancer cells harbouring KRAS, BRAF and PIK3CA mutations. PLoS One 2012; 7 (7): e41347.

17. Ogino S, Nosho K, Kirkner GJ, Shima K, Irahara N, Kure S, et al. PIK3CA mutation is associated with poor prognosis among patients with curatively resected colon cancer. J Clin Oncol 2009; 27 (9): 1477-84.

18. Liao X, Morikawa T, Lochhead P, Imamura Y, Kuchiba A, Yamauchi M, et al. Prognostic role of PIK3CA mutation in colorectal cancer: cohort study and literature review. Clin Cancer Res 2012; 18 (8): 2257-68.

19. Winder T, Lenz HJ. Molecular predictive and prognostic markers in colon cancer. Cancer Treat Rev 2010; 36 (7): 550-6.

20. Lurje G, Zhang W, Lenz HJ. Molecular prognostic markers in locally advanced colon cancer. Clin Colorectal Cancer 2007; 6 (10): 683-90.

21. Bellolio RF, Álvarez VK, De la Fuente LM, León GF, Fullerton M DA, Soto DG, et al. [Hereditary colorectal cancer: Molecular analysis of APC and MLH1 genes]. Rev Med Chile 2006; 134 (7): 841-8.

22. Torres VA, Tapia JC, Rodríguez DA, Lladser A, Arredondo C, Leyton L, et al. E-cadherin is required for caveolin-1-mediated down-regulation of the inhibitor of apoptosis protein survivin via reduced beta-cateninTcf/Lef-dependent transcription. Mol Cell Biol 2007; 27 (21): 7703-17.

23. De la Fuente MK, Álvarez KP, Letelier AJ, Bellolio F,
Acuña ML, León FS, et al. Mutational screening of the APC gene in Chilean families with familial adenomatous polyposis: nine novel truncating mutations. Dis Colon Rectum 2007; 50 (12): 2142-8.

24. Roa JC, Roa I, Melo A, Araya JC, Villaseca MA, Flores $\mathrm{M}$, et al. [p 53 gene mutation in cancer of the colon and rectum]. Rev Med Chile 2000; 128 (9): 996-1004.

25. López-Kostner F. Gene symbol: APC. Disease: Adenomatous polyposis coli. Hum Genet 2008; 124 (3): 316.

26. Roa SJC, García MP, Melo AA, Tapia EO, Villaseca HM, Araya O JC, et al. [Gene methylation patterns in digestive tumors]. Rev Med Chile 2008; 136 (4): 451-8.

27. Aravena T, Passalacqua C, Castillo Taucher S. [Molecular and genetic studies for hereditary colon cancer in two patients and their families]. Rev Med Chile 2010; 138 (12): 1530-4.

28. http://omim.org/entry/190070.

29. Levidou G, Saetta AA, Gigelou F, Karlou M, Papanastasiou P, Stamatelli A, et al. ERK/pERK expression and B-raf mutations in colon adenocarcinomas: correlation with clinicopathological characteristics. World J Surg Oncol 2012; 10: 47.

30. Makrodouli E, Oikonomou E, Koc M, Andera L, Sasazuki T, Shirasawa S, et al. BRAF and RAS oncogenes regulate Rho GTPase pathways to mediate migration and invasion properties in human colon cancer cells: a comparative study. Mol Cancer 2011; 10: 118.

31. Jancik S, Drabek J, Radzioch D, Hajduch M. Clinical relevance of KRAS in human cancers. J Biomed Biotechnol 2010; 2010: 150960.

32. Neumann J, Zeindl-Eberhart E, Kirchner T, Jung A. Frequency and type of KRAS mutations in routine diagnostic analysis of metastatic colorectal cancer. Pathol Res Pract 2009; 205 (12): 858-62.

33. Knijn N, Mekenkamp LJ, Klomp M, Vink-Borger ME, Tol J, Teerenstra S, et al. KRAS mutation analysis: a comparison between primary tumours and matched liver metastases in 305 colorectal cancer patients. Br J Cancer 2011; 104 (6): 1020-6.

34. Liebvre A, Rouleau E, Buecher B, Mitry E. [Clinical significance of BRAF mutations in colorectal cancer]. Bull Cancer 2010; 97 (12): 1441-52.

35. Voigt M, Braig F, Göthel M, Schulte A, Lamszus K, Bokemeyer C, et al. Functional dissection of the epidermal growth factor receptor epitopes targeted by panitumumab and cetuximab. Neoplasia 2012; 14 (11): 1023-31.

36. Woo J, Palmisiano N, Tester W, Leighton JC. Controversies in antiepidermal growth factor receptor therapy in metastatic colorectal cancer. Cancer 2013; 119 (11): 1941-50. 
37. Laurent-Puig P, Cayre A, Manceau G, Buc E, Bachet JB, Lecomte T, et al. Analysis of PTEN, BRAF, and EGFR status in determining benefit from cetuximab therapy in wild-type KRAS metastatic colon cancer. J Clin Oncol 2009; 27 (35): 5924-30.

38. Valtorta E, Misale S, Sartore-Bianchi A, Nagtegaal ID, Paraf F, Lauricella C, et al. KRAS gene amplification in colorectal cancer and impact on response to EGFRtargeted therapy. Int J Cancer 2013 Feb 12. doi: 10.1002/ ijc.28106.

39. Petrelli F, Borgonovo K, Cabiddu M, Ghilardi M, Barni S. Cetuximab and panitumumab in KRAS wild-type colorectal cancer: a meta-analysis. Int J Colorectal Dis 2011; 26 (7): 823-33.

40. Vale CL, Tierney JF, Fisher D, Adams RA, Kaplan R, Maughan TS, et al. Does anti-EGFR therapy improve outcome in advanced colorectal cancer? A systematic review and meta-analysis. Cancer Treat Rev 2012; 38 (6): 618-25.

41. Winder T, Mundlein A, Rhomberg S, Dirschmid K, Hartmann BL, Knauer M, et al. Different types of K-Ras mutations are conversely associated with overall survival in patients with colorectal cancer. Oncol Rep 2009; 21 (5): 1283-7.

42. Pérez-Ruiz E, Rueda A, Pereda T, Alcaide J, Bautista D, Rivas-Ruiz F, et al. Involvement of K-RAS mutations and amino acid substitutions in the survival of metastatic colorectal cancer patients. Tumour Biol 2012; 33 (6): 1829-35.

43. Imamura Y, Morikawa T, Liao X, Lochhead P, Kuchiba A, Yamauchi M, et al. Specific mutations in KRAS codons 12 and 13, and patient prognosis in 1075 BRAF wildtype colorectal cancers. Clin Cancer Res 2012; 18 (17): 4753-63.

44. Anderson SM. Laboratory methods for KRAS mutation analysis. Expert Rev Mol Diagn 2011; 11 (6): 635-42.

45. Mariani P, Lae M, Degeorges A, Cacheux W, Lappartient E, Margogne A, et al. Concordant analysis of KRAS status in primary colon carcinoma and matched metastasis. Anticancer Res 2010; 30 (10): 4229-35.

46. Han CB, Li F, Ma JT, Zou HW. Concordant KRAS mutations in primary and metastatic colorectal cancer tissue specimens: a meta-analysis and systematic review. Cancer Invest 2012; 30 (10): 741-7. 